\title{
A Novel Feature Selection with Annealing For Computer Vision And Big Data Learning
}

\author{
Bhagyashree Bhoyar, Lavanya V Maguluri \\ Department of Computer Science, Dr.D.Y.Patil Institute of Engineering and Technology Pimpri,Pune \\ Department of Computer Science, Dr.D.Y.Patil Institute of Engineering and Technology Pimpri,Pune
}

\begin{abstract}
Numerous PC vision and medical imaging issues a confronted with gaining from expansive scale datasets, with a huge number of perceptions furthermore, highlights. A novel productive learning plan that fixes a sparsity imperative by continuously expelling variables taking into account a measure and a timetable. The alluring actuality that the issue size continues dropping all through the cycles makes it especially reasonable for enormous information learning. Methodology applies nonexclusively to the advancement of any differentiable misfortune capacity, and discovers applications in relapse, order and positioning. The resultant calculations assemble variable screening into estimation and are amazingly easy to execute. It gives hypothetical assurances of joining and determination consistency. Investigates genuine and engineered information demonstrate that the proposed strategy contrasts exceptionally well and other cutting edge strategies in relapse, order and positioning while being computationally exceptionally effective and adaptable.
\end{abstract}

Keywords - MMCD, SDM, Feature Selection, Big Data, Computer Vision

Date of Submission: 17 May 2016

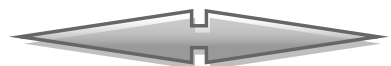

Date of Accepted: 14 November 2016

\section{INTRODUCTION}

Very popular technique of feature selection is decision for speed communication \& accuracy. For huge amounts of data required learning classifier for computer vision and imaging problems. Following are the important summons for feature selection.

1. Efficiency: In large scale computation, learning algorithm is fast and scalable is attractive.

2. Statistical guarantee: In big dataset with high portability, truthful learning approaches much recover genuine signal to pollute sound and innumerable inconvenience.

3. Universality: Instance regression and classification are the modified types of problems of universal leaning rather than limit of the problem.

4. Implementation ease: Calculations that are easy to execute can maintain a strategic distance from over-fitting and specially appointed plans. Regularization parameters ought to be characterized on account of simplicity of tuning. In some true applications, it is useful to have a calculation with adaptable cost in light of registering assets.

5. Nonlinearity: Direct blends of informative variables may not suffice in learning and displaying. Joining nonlinearity is essential in numerous huge information applications. As of late, punished techniques have gotten a considerable measure of consideration in high-dimensional element determination. They take care of a class of improvement issues with sparsity actuating punishments, for example, the L1, L0, and SCAD There is a factual surety that garbage measurements can be evacuated with high likelihood (even in high measurements) However these advancement based calculations are not sufficiently adaptable and the tuning of the punishment parameter could be tedious on vast datasets.

Feature selection improves the performance of classification algorithm - Classification algorithm may not scale up to the size of the full feature set either in sample or time and it allows us to better understand the domain

Feature Selection methods can be classified as Filters and Wrappers.

- Filter Methods include simple statistical test to determine if a feature is statistically significant for example the $\mathrm{p}$ value for a t test to determine if the null hypothesis should be accepted and the feature rejected. This does not take into account feature interactions and is generally not a much recommended way of doing feature selection as it can lead to lost in information.

- Wrapper based methods involve using a learning algorithm to report the optimal subset of features. An example would be how widely used by the competitive data science community to determine the importance of features by looking at the information gain. This can give a rather quickly and dirty overview 
of which features are important which can help provide some informal validation of engineered features. Tree based models like Random Forest are also robust against issues like multi-co linearity, missing values, outliers etc as well as being able to discover some interactions between features. However this can be rather computationally expensive.

- Boosting is used to reduce the error rate and the success of boosting depends upon on the choice of the given classifier and the principle behind this method is that although a classification algorithm may only be able to produce a model with slightly better accuracy than random guessing.

\section{LITERATURE SURVEY}

1. Zhou, Yingbo, et al. "Parallel feature selection inspired by group testing."Advances in Neural Information Processing Systems. 2014.

Feature selection is applied to reduce the number of features in many applications where data has hundreds or thousands of features. Existing feature selection methods mainly focus on finding relevant features. Feature redundancy is defined and proposed to perform explicit redundancy analysis in feature selection. Some randomized tests are performed in parallel.

2. Jiang, Dingfeng, and Jian Huang. "Majorization minimization by coordinate descent for concave penalized generalized linear models." Statistics and computing 24.5 (2014): 871-883.

Late studies have shown hypothetical engaging quality of a class of curved punishments in variable determination, including the easily cut total deviation and minimax inward punishments. The calculation of the inward punished arrangements in high-dimensional models, in any case, is a troublesome undertaking. We propose a majorization minimization by direction plummet (MMCD) calculation for processing the inward punished arrangements in summed up straight models. As opposed to the current calculations that utilization neighborhood quadratic or nearby direct guess to the punishment work, the MMCD tries to majorize the negative log-probability by a quadratic misfortune, however does not utilize any estimate to the punishment.

3. Jung, Heechul, Jeongwoo Ju, and Junmo Kim. "Rigid motion segmentation using randomized voting." Proceedings of the IEEE Conference on Computer Vision and Pattern Recognition. 2014.

Rigid motion algorithm is based on epipolar geometry, and computes a score using the distance between the feature point and the corresponding epipolar line. This score is accumulated and utilized for final grouping. It is also called as Randomized voting (RV) algorithm and it is based on epipolar geometry, and computes a score using the distance between the feature point and the corresponding epipolar line. This score is accumulated and utilized for final groupin and it basically deals with two frames, so it is also applicable to the two-view motion segmentation problem.

4. Xiong, Xuehan, and Fernando Torre. "Supervised descent method and its applications to face alignment." Proceedings of the IEEE conference on computer vision and pattern recognition. 2013.

Numerous PC vision issues (e.g., camera adjustment, picture arrangement, structure from movement) are illuminated through a nonlinear advancement strategy. It is for the most part acknowledged that 2 nd request plunge strategies are the most hearty, quick and dependable methodologies for nonlinear improvement of a general smooth capacity. Be that as it may, with regards to PC vision, 2 nd request plunge techniques have two fundamental downsides: (1) The capacity won't not be logically differentiable and numerical approximations are unfeasible. (2) The Hessian may be huge and not positive unequivocal. To address these issues, this proposes an Administered Drop Strategy (SDM) for minimizing a Non-direct Slightest Squares (NLS) capacity.

5. Sun, Yi, Xiao gang Wang, and Xiaoou Tang. "Deep convolution network cascade for facial point detection." Proceedings of the IEEE Conference on Computer Vision and Pattern Recognition. 2013.

Another methodology for estimation of the positions of facial key focuses with three-level painstakingly outlined convolution systems. At every level, the yields of numerous systems are intertwined for vigorous and exact estimation. On account of the profound structures of convolution systems, worldwide abnormal state elements are removed over the entire face district at the introduction stage, which find high exactness key focuses. There are two folds of favorable position for this The strategy accordingly can keep away from nearby least brought on by vagueness and information defilement in troublesome picture tests because of impediments, vast posture varieties, and great lightings.

6. She, Yiyuan. "An iterative algorithm for fitting nonconvex penalized generalized linear models with grouped predictors." Computational Statistics \& Data Analysis 56.10 (2012): 2976-2990. 
High-dimensional information posture challenges in measurable learning and demonstrating. Here and there the indicators can be actually assembled where seeking after the between-gathering sparsity is sought. Collinearity may happen in true high-dimensional applications where the well known method experiences both choice irregularity and forecast incorrectness. In addition, the issues of interest frequently go past Gaussian models.. A thorough hypothetical result ensures its meeting and gives tight preparatory scaling.

7. Ding, Liangjing, Adrian Barbu, and Anke Meyer-Baese. "Motion segmentation by velocity clustering with estimation of subspace dimension." Computer Vision-ACCV 2012 Workshops. Springer Berlin Heidelberg, 2012.

The execution of bunching construct movement division strategies depends with respect to the measurement of the subspace where the point directions are anticipated. This paper introduces a methodology for evaluating the best subspace measurement utilizing a novel grouping blunder measure. For each got division, the proposed measure appraises the normal minimum square mistake between the point directions and engineered directions created in view of the movement models from the division.

8. Köstinger, Martin, et al. "Annotated facial landmarks in the wild: A large-scale, real-world database for facial landmark localization." Computer Vision Workshops (ICCV Workshops), 2011 IEEE International Conference on. IEEE, 2011.

Face arrangement is a pivotal stride in face acknowledgment errands. Particularly, utilizing historic point restriction for geometric face standardization has appeared to be extremely successful, plainly enhancing the acknowledgment results. Notwithstanding, no satisfactory databases exist that give an adequate number of commented on facial points of interest. The databases are either constrained to frontal perspectives, give just a little number of clarified pictures or have been procured under controlled conditions. Subsequently, we present a novel database defeating these impediments:

9. Breheny, Patrick, and Jian Huang. "Coordinate descent algorithms for nonconvex penalized regression, with applications to biological feature selection." The annals of applied statistics 5.1 (2011): 232.

Various variable determination strategies have been proposed including non curved punishment capacities. These techniques, which incorporate the easily cut total deviation (SCAD) punishment and the minima sunken punishment (MCP), have been shown to have appealing hypothetical properties, however display fitting is not a clear undertaking, and the subsequent arrangements might be flimsy. Moreover, show the utility of convexity diagnostics to decide districts of the parameter space in which the target capacity is locally raised, despite the fact that the punishment is definitely not.

III. RELATED WORK

\begin{tabular}{|l|l|l|l|}
\hline REF.NO & ALGORITHM & ADVANTAGES & DISADVANTAGES \\
\hline REF NO[2] & MMCD & $\begin{array}{l}\text { MMCD to logistic relapse is encouraged by } \\
\text { the way that a basic and powerful } \\
\text { majorization capacity can be built for the } \\
\text { logistic }\end{array}$ & $\begin{array}{l}\text { In models in the GLM family, for example, } \\
\text { the log-straight model, it creates the } \\
\text { impression that no basic majorization } \\
\text { capacity exists. }\end{array}$ \\
\hline REF NO[3] & RV & $\begin{array}{l}\text { It accomplished inside a reason capable time, } \\
\text { the most noteworthy execution of all other } \\
\text { best in class calculations, furthermore } \\
\text { accomplished practically identical exactness } \\
\text { under boisterous natural conditions }\end{array}$ & accuracy decreases \\
\hline REF NO[4] & $\begin{array}{l}\text { PAM } \\
\text { ALGORITHM }\end{array}$ & $\begin{array}{l}\text { Approach in the minimization of explanatory } \\
\text { capacities, and in the issue of facial element } \\
\text { discovery }\end{array}$ & $\begin{array}{l}\text { Hard to apply the SDM to different NLS in } \\
\text { PC vision, for example, camera alignment } \\
\text { and structure from movement. }\end{array}$ \\
\hline REF NO[5] & $\begin{array}{l}\text { SVM } \\
\text { RANDOM } \\
\text { FOREST }\end{array}$ & $\begin{array}{l}\text { To estimate of the positions of facial key } \\
\text { points with three-level carefully designed } \\
\text { convolution networks }\end{array}$ & $\begin{array}{l}\text { powerful classifiers or repressors, are } \\
\text { required as the visual complexity increases } \\
\text { exponentially with the size of the image } \\
\text { region }\end{array}$ \\
\hline REF NO[7] & KNN & $\begin{array}{l}\text { for estimating the best subspace dimension } \\
\text { using a novel clustering error measure }\end{array}$ & $\begin{array}{l}\text { need to determine the value of the } \\
\text { parameter for the nearest neighbors }\end{array}$ \\
\hline REF NO[9] & $\begin{array}{l}\text { LARS } \\
\text { for fitting linear regression models to high- } \\
\text { dimensional data }\end{array}$ & $\begin{array}{l}\text { There may be no reason to believe that the } \\
\text { selected variables will have a high } \\
\text { probability of being the actual underlying } \\
\text { causal variables. }\end{array}$ \\
\hline
\end{tabular}

\section{CONCLUSION}

A novel learning plan for highlight determination is high dimensional information applications. It continuously recognizes and expels some immaterial variables and continues as per a toughening plan. It takes 
care of a compelled advancement issue and has an execution ensure in both estimation and determination. The calculation is reasonable for huge information calculation because of its straightforwardness and capacity to diminish the issue size all through the emphasis.. Henceforth in calculation, FSA has comparative points of interest as an online calculation (that gets to every preparation perception once) while being significantly more exact. Approach applies blandly excessively numerous sorts of issues, including relapse, order and positioning for example. Broad analyses on both manufactured information and genuine information bolster FSA as an aggressive contrasting option to numerous up and coming element choice strategies. Later on it will apply the variable determination strategy to testing object recognition issues.

\section{REFERENCES}

[1]. Zhou, Yingbo, et al. "Parallel feature selection inspired by group testing."Advances in Neural Information Processing Systems. 2014.

[2]. Jiang, Dingfeng, and Jian Huang. "Majorization minimization by coordinate descent for concave penalized generalized linear models." Statistics and computing 24.5 (2014): 871-883

[3]. Jung, Heechul, Jeongwoo Ju, and Junmo Kim. "Rigid motion segmentation using randomized voting." Proceedings of the IEEE Conference on Computer Vision and Pattern Recognition. 2014.

[4]. Xiong, Xuehan, and Fernando Torre. "Supervised descent method and its applications to face alignment." Proceedings of the IEEE conference on computer vision and pattern recognition. 2013

[5]. Sun, Yi, Xiao gang Wang, and Xiaoou Tang. "Deep convolution network cascade for facial point detection." Proceedings of the IEEE Conference on Computer Vision and Pattern Recognition. 2013.

[6]. Breheny, Patrick, and Jian Huang. "Coordinate descent algorithms for nonconvex penalized regression, with applications to biological feature selection." The annals of applied statistics 5.1 (2011): 232

[7]. She, Yiyuan. "An iterative algorithm for fitting nonconvex penalized generalized linear models with grouped predictors." Computational Statistics \& Data Analysis 56.10 (2012): 2976-2990.

[8]. Ding, Liangjing, Adrian Barbu, and Anke Meyer-Baese. "Motion segmentation by velocity clustering with estimation of subspace dimension." Computer Vision-ACCV 2012 Workshops. Springer Berlin Heidelberg, 2012.

[9]. Breheny, Patrick, and Jian Huang. "Coordinate descent algorithms for nonconvex penalized regression, with applications to biological feature selection." The annals of applied statistics 5.1 (2011): 232 